\title{
Nanofabricated Neural Probes for Dense 3-D Recordings of Brain Activity
}

\author{
Gustavo Rios, ${ }^{\dagger}$ Evgueniy V. Lubenov, ${ }^{\dagger}$ Derrick Chi, ${ }^{\S}$ Michael L. Roukes, ${ }^{*}, \dagger, \ddagger, \S, \|$ and \\ Athanassios G. Siapas*, $*$
}

${ }^{\dagger}$ Division of Biology and Biological Engineering, ${ }^{\ddagger}$ Division of Engineering and Applied Science, ${ }^{\S}$ Division of Physics, Mathematics, and Astronomy, and "Kavli Nanoscience Institute, California Institute of Technology, Pasadena, California 91125, United States

\section{Supporting Information}

ABSTRACT: Computations in brain circuits involve the coordinated activation of large populations of neurons distributed across brain areas. However, monitoring neuronal activity in the brain of intact animals with high temporal and spatial resolution has remained a technological challenge. Here we address this challenge by developing dense, threedimensional (3-D) electrode arrays for electrophysiology. The 3-D arrays constitute the front-end of a modular and configurable system architecture that enables monitoring neuronal activity with unprecedented scale and resolution.
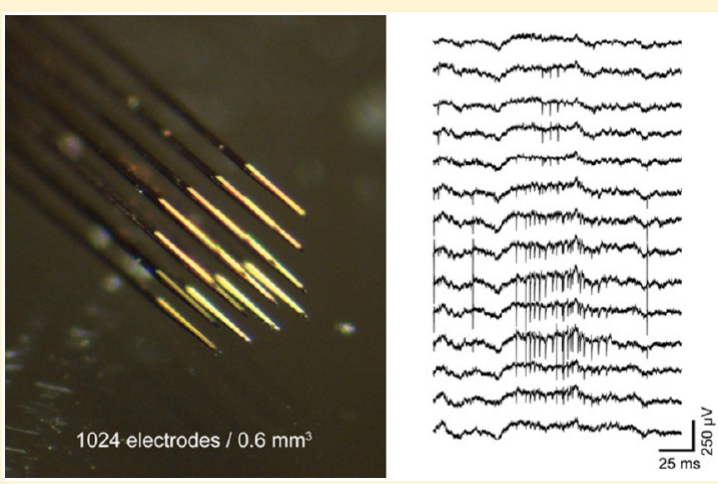

KEYWORDS: Nanofabricated neural probes, high-density microelectrodes, 3-D scalable packaging, brain mapping

$\mathrm{B}$ rain functions such as perception, motor control, learning, and memory arise from the coordinated activation of neuronal assemblies distributed across multiple brain areas. While

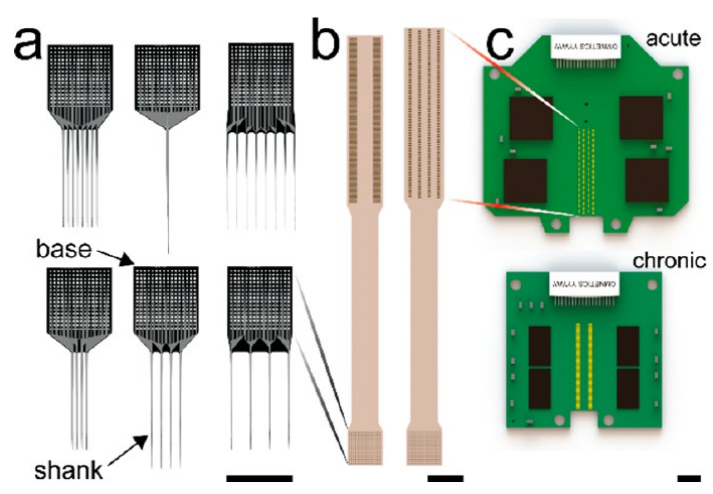

Figure 1. Recording system modules. (a) Examples of six realized neural probe designs. The number of shanks (1-8), intershank spacing $(250-1000 \mu \mathrm{m})$, recording site arrangement, and pitch (20$65 \mu \mathrm{m})$ are configurable. All designs support 256 electrodes per layer, connected to a standard $16 \times 16$ interconnect matrix with $200 \mu \mathrm{m}$ pitch at the probe base. (b) Designs of two ultraflexible cables (fabricated on either $10 \mu \mathrm{m}$ thick Parylene $\mathrm{C}$ or $15 \mu \mathrm{m}$ thick polyimide) used to interface the neural probes to the signal conditioning PCB. (c) Designs of two different signal conditioning PCBs (headstages). Each performs analog signal conditioning, multiplexing, and digitization of 256 analog inputs. The top circuit (acute) measures $39 \times 37 \mathrm{~mm}$, weighs $4.5 \mathrm{~g}$, employs 8 Intan RHD2132 QFN packaged chips, and requires 8 output LVDS lines (16 wires), while the bottom circuit (chronic) measures $30 \times 32 \mathrm{~mm}$, weighs $1.2 \mathrm{~g}$, uses 4 Intan RHD2164 bare dies, and requires four output LVDS lines (eight wires). (a-c) Scale bar: $3.4 \mathrm{~mm}$. major progress has been made in understanding the response properties of individual cells, circuit interactions remain poorly understood. One of the fundamental obstacles to understanding these interactions has been the difficulty of measuring the activity of large distributed populations of neurons in behaving animals. ${ }^{1-5}$ Electrophysiology has been the gold standard for monitoring the brain because it measures the electrical activity of neurons directly and at a high temporal resolution, sufficient to capture in detail even the fastest neuronal events. The main drawback of electrophysiology has been the invasiveness of the recording electrodes and the consequent limits on the spatial extent and spatial resolution of the obtained signals.

Research on electrical probes has focused on overcoming these challenges by scaling up the number of recording sites while minimizing their invasiveness. ${ }^{1,4,6-15}$ These are inherently competing objectives because smaller probes, with mechanical dimensions that minimize tissue displacement, offer less surface area and volume for electrode sites, interconnects, and active circuit elements. ${ }^{16}$ Furthermore, as electrode count increases, so does the need to bring active signal conditioning and multiplexing components closer to the brain, as the number of passive interconnects exceeds the limits of connector and tether cable density. This, in turn, introduces another dimension to the invasiveness of the recording system-the amount of electrical power it dissipates as heat into the brain tissue. Chronic viability of the probes imposes additional constraints

Received: June 28, 2016

Revised: September 17, 2016

Published: October 21, 2016 
a
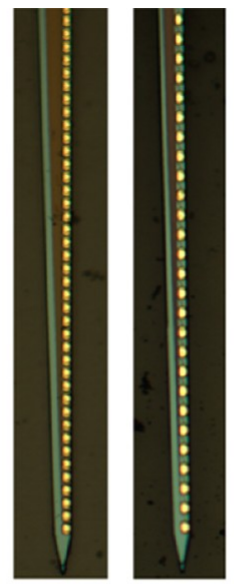

$20 \mu \mathrm{m} \quad 30 \mu \mathrm{m}$

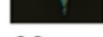

$60 \mu \mathrm{m}$

g

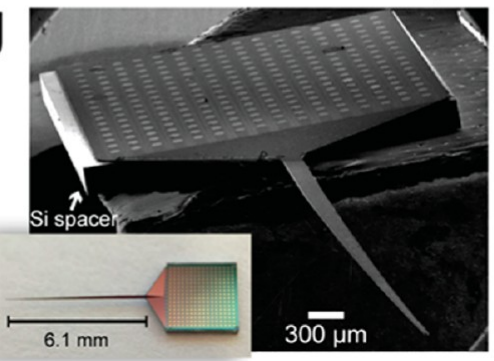

b
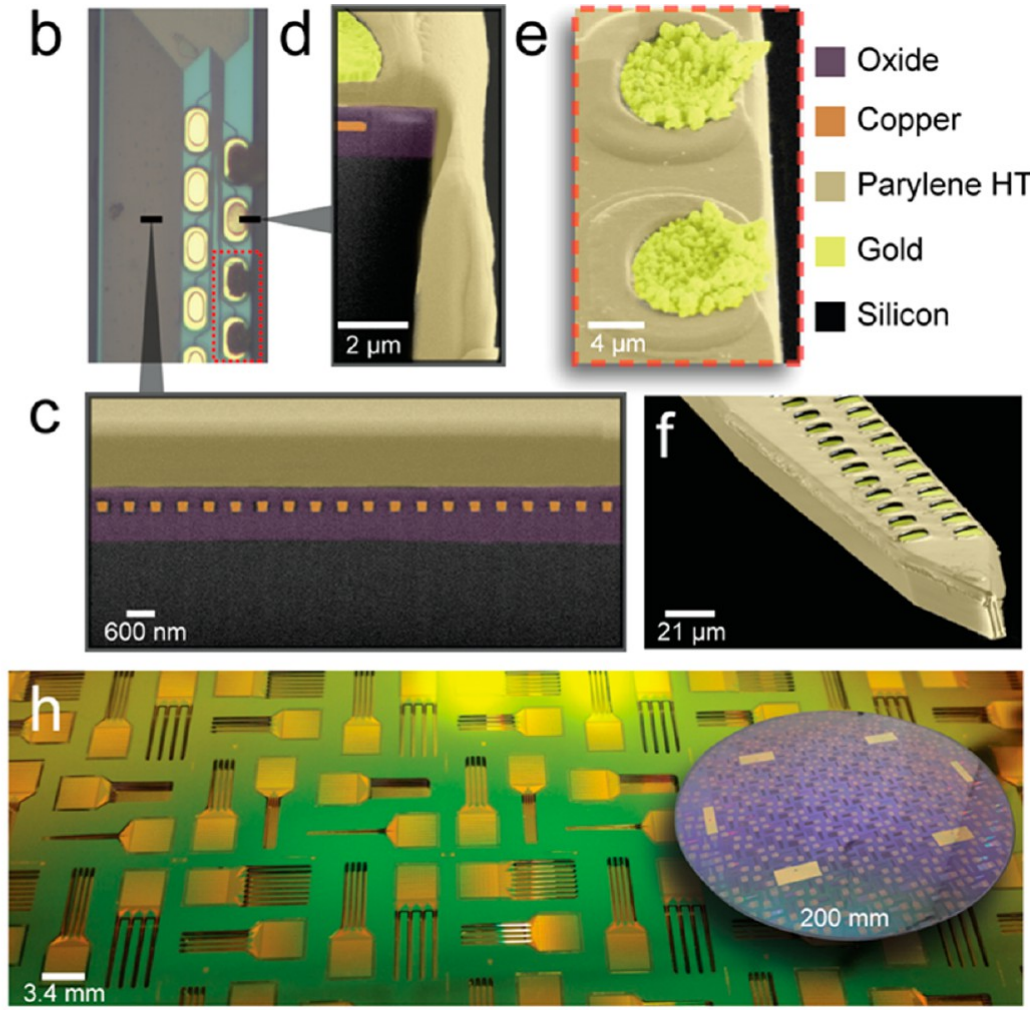

Figure 2. Minimally invasive high-density neural probes. (a) Microscope images of four different shank tips with different electrode configurations. The shank width at the electrode furthest from the tip is less than $65 \mu \mathrm{m}$ for all but one design, shown in g, while shank width at the base is $100 \mu \mathrm{m}$. (b) Shank width is minimized by using nanoscale interconnects. Shank areas subjected to sectioning by focused ion beam (FIB) milling are marked with black lines, and the red rectangle marks a region imaged with scanning electron microscopy (SEM). (c-f) False color SEMs indicating different materials according to color legend on the right. (c) Shank cross section reveals nanoscale $(300 \times 300 \mathrm{~nm})$ copper interconnects (orange) with a pitch of $600 \mathrm{~nm}$ buried in $1.6 \mu \mathrm{m}$ of oxide insulation (purple, see Supplementary Figure S1 for details). (d) Cross section at the shank edge demonstrates conformal coverage of shank sidewall by a biocompatible Parylene HT layer (tan). (e) Two gold electroplated microelectrodes (yellow) demonstrate the increase in electrode surface area and roughness while preserving planar dimensions. (f) Tip of the $21 \mu \mathrm{m}$ thick shank demonstrates conformal coverage of three sides (top and sidewalls) by Parylene HT (tan). The bottom side of the shank is composed of 900 nm $\mathrm{SiO}_{2}$, which is also biologically inert. (g) SEM and stereoscope (inset) image of a probe mounted on a slightly wider silicon spacer. The probe thickness is $21 \mu \mathrm{m}$ throughout and can be assembled onto a spacer of arbitrary thickness to control the pitch of a $3 \mathrm{D}$ stack (300 $\mu \mathrm{m}$ thick spacer shown). (h) Devices fabricated at a commercial foundry (LETI, Grenoble, France) on $200 \mathrm{~mm}$ SOI wafers (inset) are fully released and anchored in place on an SOI wafer.

on the biocompatibility of all materials that come in direct contact with brain tissue as well as on the flexibility of the probe itself and its coupling to the rest of the system. ${ }^{17-21}$ Finally, relating the measured extracellular potentials to the underlying circuit elements requires solving an inverse problem to obtain a detailed current source density (CSD) distribution. ${ }^{22}$ The quality of this CSD estimate critically depends on the density of electrodes and their three-dimensional (3-D) arrangement on a regular array of known dimensions and relative position to the tissue. ${ }^{4}$ While significant progress has been made in solving the above issues individually, addressing them simultaneously within a full system has remained a challenge.

Here we describe the development of a modular, scalable system for dense 3-D chronic electrophysiology that addresses many of the challenges above. The front end of the system is comprised of passive high-density nanofabricated neural probes (nanoprobes, Figure 1a) - 2-D arrays of minimally invasive shanks with nanoscale interconnects-that are subsequently stacked into a 3-D array of precise geometry with over a thousand recording sites. The front end of the system is mechanically and thermally decoupled from all active components through high-density flexible cables (Figure 1b), which interface the neural probes to the signal conditioning, multiplexing, and digitizing circuitry. The latter is housed on compact, lightweight PCBs (Sierra Circuits, HDI PCB technology), compatible with acute and chronic experimentation (Figure 1c). We describe the design, fabrication, and assembly of the system and its performance characteristics. We also demonstrate the realized yield and quality of electrophysiological recordings in experiments with awake head-fixed mice.

Each neural probe is a thin $(21 \mu \mathrm{m})$ silicon device with a square base $(3.4 \times 3.4 \mathrm{~mm})$ and up to eight narrow $(65 \mu \mathrm{m})$ shanks containing a total of 256 microelectrode sites $(8 \times$ $16 \mu \mathrm{m}$ ovals) distributed in single or double row configurations (Figures 1-2). The base houses a $16 \times 16$ interface matrix of $100 \mu \mathrm{m}$ circular pads with $100 \mu \mathrm{m}$ edge-to-edge spacing, which constitutes the standardized interface between the probe and the rest of the system (Figure 1a). In order to minimize the invasiveness of the shanks, while maintaining high electrode site density, the following design choices were implemented. First, the width of shanks was kept at a minimum in order to reduce mechanical invasiveness through tissue displacement. ${ }^{23}$ In all but one design, the maximal shank width in the span containing electrodes was less than $65 \mu \mathrm{m}$ (50 $\mu \mathrm{m}$ on average), while shanks 

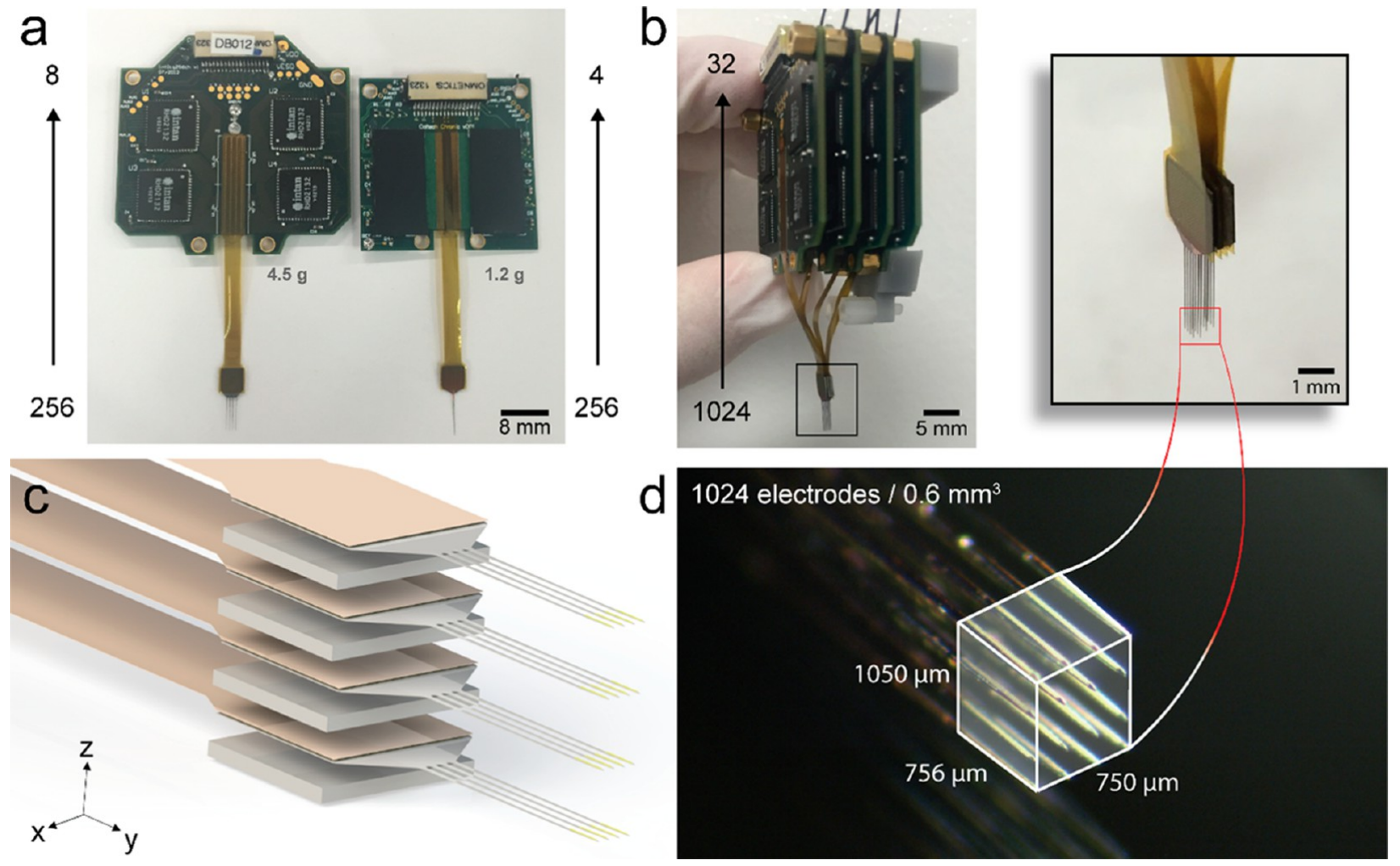

Figure 3. Recording modules configured as a 3-D array with 1024 electrodes. (a) Acute (left) and chronic (right) 256-channel recording modules consisting of a neural probe, flexible cable, and signal conditioning PCB. (b) Four recording modules are assembled as layers into a stack to form a 1024-electrode 3-D array (system weight $20 \mathrm{~g}$, including $3 \mathrm{~mm}$ tall PCB brass spacers; chronic system weight $6.8 \mathrm{~g}$ ). (right) Close-up view of the stacked neural probes. (c) 3-D electrode array is highly compact and configurable. The shank spacing of the selected neural probe controls electrode pitch along the $x$-axis, with available options ranging from $250 \mu \mathrm{m}$ to $1 \mathrm{~mm}$. Electrode spacing along the shanks of the selected neural probe controls pitch along the $y$-axis, with available options ranging from 12 to $65 \mu \mathrm{m}$. The silicon spacer thickness (arbitrary) controls the electrode pitch along the $z$-axis. A minimum $z$-pitch of $50 \mu \mathrm{m}$, which can be achieved without the use of the spacer, is determined by the combined thickness of the neural probe base $(21 \mu \mathrm{m})$, ACF $(14 \mu \mathrm{m})$, and flexible cable $(15 \mu \mathrm{m})$. (d) The 3D electrode array used to obtain in vivo recordings. Its $x-y-z$ pitch is $250-12-350 \mu \mathrm{m}$, and the volume enclosed by the array is $750-756-1050 \mu \mathrm{m}$, giving an electrode density of 1024 electrodes for $0.6 \mathrm{~mm}^{3}$.

where much narrower near the tip $(24 \mu \mathrm{m})$ and only gradually widened to about $100 \mu \mathrm{m}$ near the probe base (Figure $2 \mathrm{a}$ ). Narrow shanks were made possible by utilizing nanoscale interconnects, which had a $300 \times 300 \mathrm{~nm}$ cross-section and were spaced at $300 \mathrm{~nm}$ (Figure 2c). Second, electrodes were small in area $\left(117 \mu \mathrm{m}^{2}\right)$ and shaped as ovals elongated parallel to the shank axis $(8 \times 16 \mu \mathrm{m})$, which further minimized the shank width (Figure 2b). Low impedance was achieved in this small microelectrode area by gold electrodeposition), which increases the effective electrode surface area without altering its planar dimensions (Figure 2e). ${ }^{24,25}$ Third, shanks were coated with a Parylene HT biocompatibility layer ${ }^{26}$ on 3 sides, while the backside was made of biologically inert glass (silicon oxide) (Figure 2d,f). Fourth, the probes are completely passive devices interfaced to all powered electronics through a $15 \mu \mathrm{m}$ thin ultraflexible cable (Metrigraphics LLC), which isolates the probes both thermally and mechanically from the rest of the system. Finally, while all devices were developed in-house on $100 \mathrm{~mm}$ SOI wafers using electron beam lithography and MEMS fabrication procedures (Kavli Nanoscience Institute, Caltech), the final probes were nanofabricated using a hybrid CMOS/MEMS process on $200 \mathrm{~mm}$ SOI wafers at a commercial state-of-the-art semiconductor foundry (LETI, Grenoble, France; see Supplementary Figure S1 for details). This improved device yield, quality, and consistency (Figure $2 \mathrm{~h}$ ).
While recent work has highlighted the potential advantages that more flexible substrates may provide, ${ }^{27-31}$ we fabricated the neural probes using silicon on isolator (SOI) wafers with thin $(17 \mu \mathrm{m})$ device layer in order to guarantee precise and reproducible three-dimensional (3-D) electrode arrangements (Figure 3). Mechanical decoupling of the probe was achieved by interfacing it to the rest of the system using ultraflexible cables. The probe, cable, and PCB were flip-chip bonded together (Fineplacer Lambda, Finetech) using the anisotropic conductive film (ACF, H\&S Hightech, TCF1051GY for probe to flex cable bond; TGP2050N for flex cable to PCB bond) to produce a fully functional 2-D recording module (Figure $3 \mathrm{a}$ ). The use of ACF was essential for accomplishing low contact resistance $(<1 \Omega)$ connections within the compact, fine-pitched probe pad matrix. The 2-D modules were used as layers that were then combined together into the 3-D stack (Figure 3b). The neural probes comprising the 3-D electrode array were precisely aligned with the flip-chip bonder, spaced using silicon spacers of $300 \mu \mathrm{m}$ thickness (Figure $2 \mathrm{~g}$ ), and bonded together with either polyethylene glycol (PEG, MW: 3000, Sigma; temporary bond) or thin epoxy sheets (AiT Technology, ESP8680-HF; permanent bond; Figure 3c). Notice that the 3-D electrode array is highly configurable through choice of neural probe model, spacer thickness, and probe alignment. To demonstrate the power of this approach we assembled a dense 3-D electrode array with 1024 electrodes spanning a $0.6 \mathrm{~mm}^{3}$ volume (Figure $3 \mathrm{~d}$ ). 

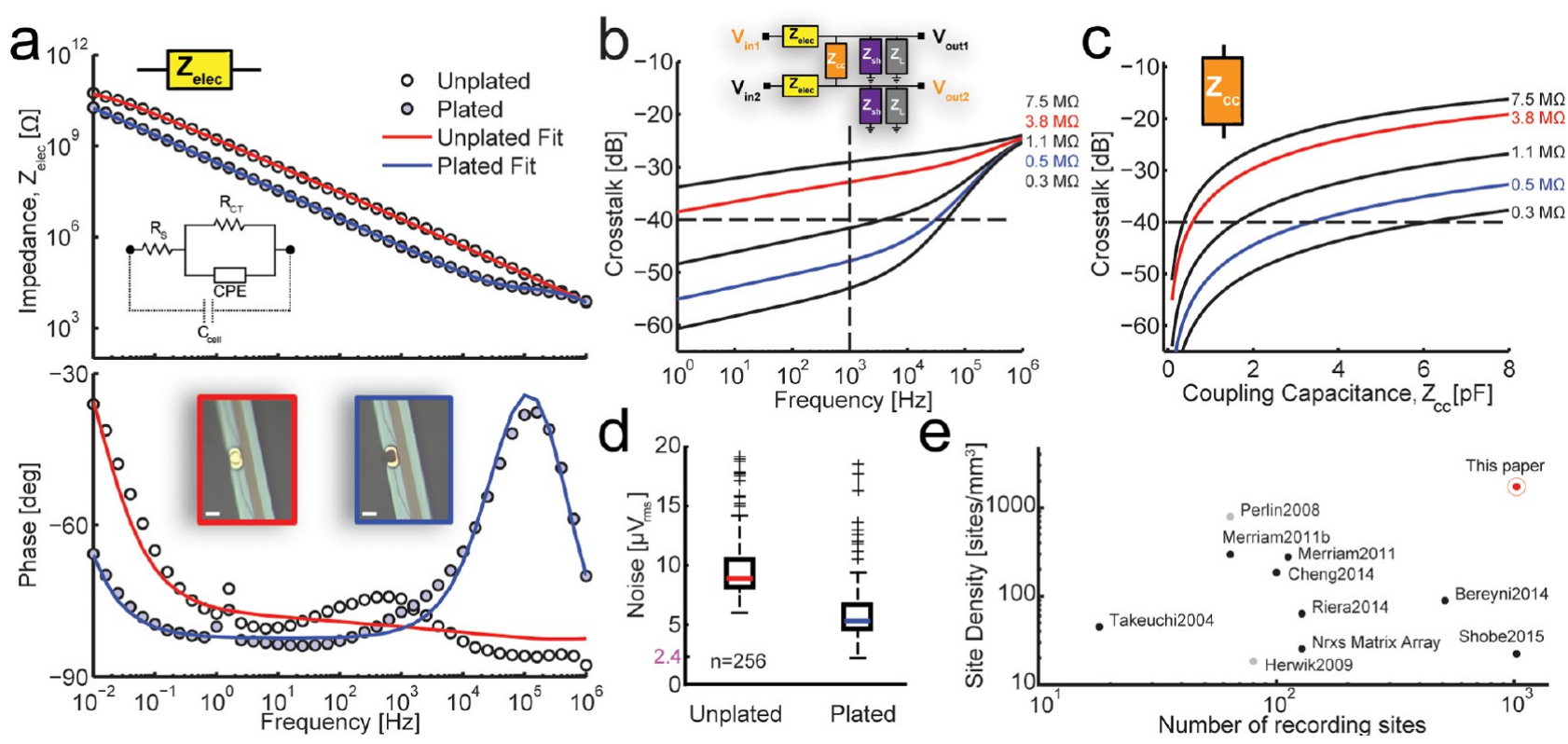

Figure 4. System characteristics and comparison to other 3-D neural recording systems. (a) Equivalent circuit model (top inset) for an unplated (red) and gold-plated (blue) electrode-electrolyte interface derived from electrochemical impedance spectroscopy (EIS) data, displayed as Bode plot and captured using a fully passive assembly. Equivalent circuit parameters for unplated (plated) electrodes were: spreading resistance $R_{\mathrm{s}}=20 \mathrm{k} \Omega$ $(15 \mathrm{k} \Omega)$, charge transfer resistance $R_{\mathrm{ct}}=55 \mathrm{G} \Omega(89 \mathrm{G} \Omega)$, constant phase element (CPE) exponent $\alpha=0.88(0.91)$, CPE prefactor $Q=60 \times 10^{-12}$ $\left(750 \times 10^{-12}\right) \mathrm{s}^{\alpha / \Omega}$, resulting effective capacitance $C_{\mathrm{e}}=9.4 \mathrm{pF}(243 \mathrm{pF}) . C_{\text {cell }}$ is the parasitic capacitance introduced by the measurement setup, $C_{\text {cell }}=12 \mathrm{pF}$. Notice that gold electroplating reduces the electrode impedance by an order of magnitude (from $3.8 \mathrm{M} \Omega$ to $500 \mathrm{k} \Omega$ at $1 \mathrm{kHz}$ ) due to a corresponding increase in the electrode's effective double layer capacitance. Microscope images (bottom inset) of an unplated (red) and plated (blue) electrode. Scale bar: $8 \mu \mathrm{m}$. (b) Equivalent circuit model (inset) used to analyze crosstalk between two adjacent interconnects. ${ }^{10}$ Traces correspond to increasing electrode impedance (values at $1 \mathrm{kHz}$ shown on right) while all remaining parameters are kept constant at values estimated for our system (coupling capacitance between adjacent traces, $C_{\mathrm{ss}}=1.35 \mathrm{pF}$, trace shunting capacitance to ground, $C_{\mathrm{sh}}=2.5 \mathrm{pF}$, amplifier input capacitance $C_{\mathrm{L}}=12 \mathrm{pF}$ ). (c) Cross-talk at $1 \mathrm{kHz}$ for increasing values of the coupling capacitance (traces as in b). Notice that, even for low impedance electrodes, a coupling capacitance above $8 \mathrm{pF}$ results in crosstalk in excess of $1 \%$. (d) System noise (RMS) of unplated (left, $9.4 \mu \mathrm{V}$ median) and plated (right, $4.8 \mu \mathrm{V}$ median) microelectrodes (bandwidth: $0.1 \mathrm{~Hz}$ to $7.5 \mathrm{kHz}$ ). The input referred noise of the amplifier is $2.4 \mu \mathrm{V}$. (e) Electrode count $(1024)$ and density $\left(1720 \mathrm{el} / \mathrm{mm}^{3}\right)$ of our realized 3-D array in comparison with previous work.

Our system architecture separates the active signal conditioning circuits from the neural probe to minimize heat dissipation from the active electronics into the brain (see Supplementary Figure S3 for details). This requires careful budgeting of parasitic capacitances and electrode impedances in the overall system design (Figure 4). Cross-talk between adjacent traces grows with electrode impedance (Figure 4b), so we used gold electrodeposition to increase the effective microelectrode surface area thereby lowering impedance by an order of magnitude (Figure 4a). The electrochemical impedance spectra obtained before and after plating allowed us to estimate the parameters of the equivalent circuit representing the electrode-electrolyte interface (Figure 4a) and to map out the crosstalk dependence on frequency and electrode characteristics (Figure 4b). This analysis demonstrates that electrode impedance below $0.5 \mathrm{M} \Omega(0.3 \mathrm{M} \Omega)$ at $1 \mathrm{kHz}$ limits cross-talk to values below $1 \%$ for all frequencies below $1 \mathrm{kHz}(10 \mathrm{kHz})$, respectively. This range of microelectrode impedance values could be readily achieved by gold electrodeposition. Our analysis of the impact of coupling capacitance between adjacent traces on cross-talk (Figure 4c) influenced our design choice of the nanoscale interconnect cross-section, spacing, and total length. With these considerations, we achieved low cross-talk and system noise of $4.8 \mu \mathrm{V}(9.4 \mu \mathrm{V})$ RMS measured in saline for plated (unplated) electrodes, respectively (Figure $4 \mathrm{~d}$ ), in a 3-D electrode array with unprecedented density (Figure 4e).

In order to experimentally validate the system, we recorded electrophysiological activity from the hippocampus of awake, head-fixed mice, using the 1024 electrode 3-D array (Figure 5, see Supplementary Figure S2 for data acquisition details and Figure S4 for experiment setup details). The raw broadband extracellular signal from one layer of the 3-D stack is shown in Figure 5a. Notice that the low frequencies of the broadband signal, known as the local field potential (LFP), display clear and systematic spatiotemporal variations, which are a prominent and recognizable feature of hippocampal activity (Supplementary Figures S6 and S7). In contrast, the high frequency band contains high amplitude spikes, spatially restricted to nearby microelectrodes that were anatomically close to the pyramidal cell layer (Figure 5a,b). Furthermore, the same spikes are clearly seen on multiple neighboring recording sites, thereby allowing for successful triangulation of the source neuron and spike sorting (Figure 5b, Supplementary Figure S8). Although, the relative location of the 3-D electrode array with respect to the hippocampal circuitry can be inferred from the recorded patterns of electrophysiological activity alone, we directly verified it through histological sectioning and analysis (Figure 5c, Supplementary Figure S5).

One key objective of brain activity mapping is the ability to observe the firing of all neurons within a brain volume. How close does the dense 3-D electrode array described here bring us to achieving this ultimate goal? Because action potential amplitudes decay rapidly in the extracellular space, each site can only detect spikes originating within a sphere of radius $R \sim 100-150 \mu \mathrm{m}$, centered at the electrode. The union of these spheres, one for each electrode site, gives the observable 

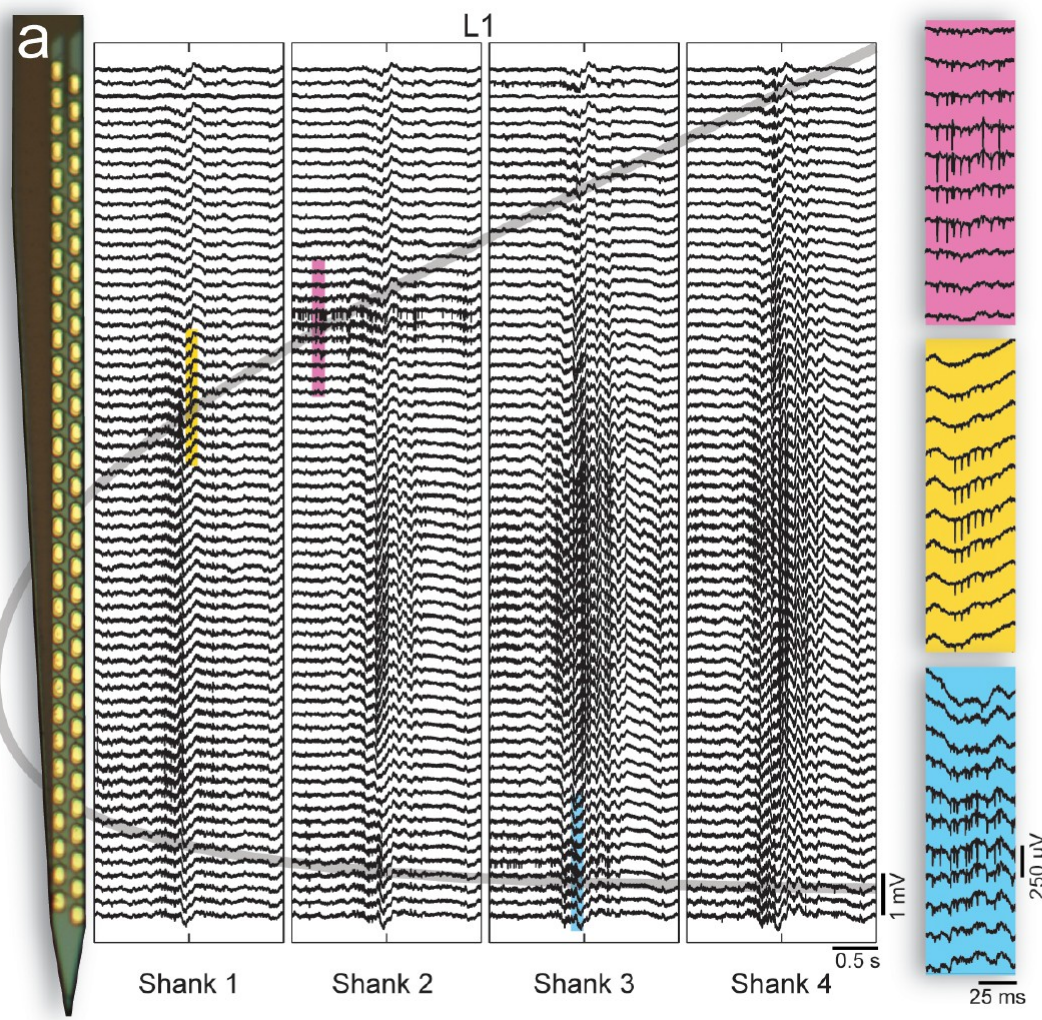
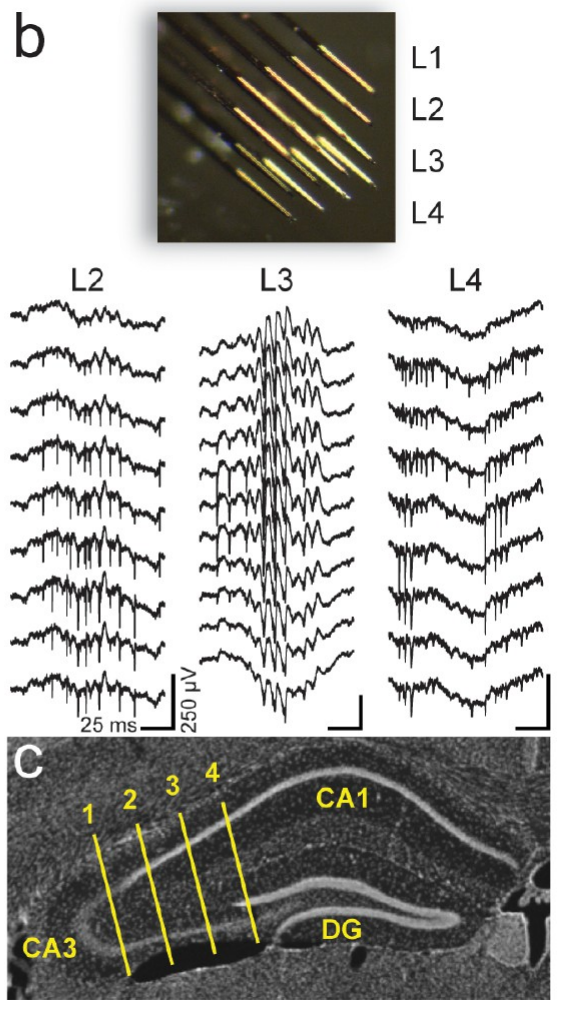

Figure 5. In vivo electrophysiological recordings using a 3-D array with 1024 electrodes. (a) Broadband signal $(0.1 \mathrm{~Hz}-7.5 \mathrm{kHz})$ from the hippocampus of an awake mouse from 4 of the 16 identical shanks (left) comprising the 3-D array. Each column displays $2 \mathrm{~s}$ of data from a single shank with traces ordered by the depth of the corresponding microelectrode site. Notice that the spatiotemporal structure in the signal reflects the anatomy and activation of the underlying circuit (cell layer marked by gray line). High-amplitude spikes are clearly visible on sites close to the cell layer (pink, orange, and blue insets). (b) Similar spiking activity is seen throughout the array for sites near the pyramidal cell layer. (c) Histological section showing the location of the shanks in panel a.

volume. The fraction of observable neurons can then be estimated as the ratio of the observable volume to the total array volume. For the dense 3-D array, this ratio initially grows approximately as the second power of the sphere radius and the estimated fraction of observable neurons is $42 \%(60 \%)$ for $R=$ $100 \mu \mathrm{m}(125 \mu \mathrm{m})$, respectively. In contrast, the volume of tissue displaced by the array is less than $1 \%$.

The ability to detect spikes is only a necessary condition for successfully isolating the firing of a source neuron. In addition, spikes from the source neuron should be detected with sufficient amplitude on several sites simultaneously. In other words, we need to consider spheres of smaller radius $R \sim 50-$ $100 \mu \mathrm{m}$ and only count the volume of overlap. Based on these considerations we estimate the fraction of resolvable neurons to be $13 \%(26 \%)$ for $R=50 \mu \mathrm{m}(75 \mu \mathrm{m})$, respectively. These numbers critically depend on the high degree of sphere overlap achieved by packing electrodes very densely $(20-24 \mu \mathrm{m})$ along the shanks, which was only possible through the use of nanoscale interconnects.

In summary, we describe the design, construction, and validation of a configurable system for dense 3-D electrophysiology. While the in vivo experiments presented above establish the proper operation of the system, significant additional work remains to fully characterize the quality of spike sorting, the yield of identifiable single units, the merit of current source density (CSD) estimates, and the long-term performance of the system under chronic conditions. These are all areas of significant theoretical and experimental interest, and the technology presented here will likely accelerate progress in these domains. For example, the highly spatially resolved electrophysiological recordings can be leveraged to analyze and improve spike sorting and CSD estimation procedures. In particular, the dense data can first be spatially subsampled to mimic common recording configurations, and then the algorithm performance on the coarsened data can be evaluated against the full set of observations. Such cross-validation approaches can be used to tune algorithm parameters and place bounds on error rates, thereby mitigating the scarcity of ground truth data.

The technology itself can be further improved by scaling up the number of recording sites by up to an order of magnitude, while maintaining the modular architecture and small displacement volume of the arrays. This would require the use of multiple interconnect layers on the shanks, denser interface matrices at the probe base, multilayer flexible cables, and higher channel count signal conditioning ASICs. Since all of these requirements effectively bring traces closer together, the parasitic capacitance budget is likely to be exhausted first. ${ }^{16}$ Beyond this point, active components will have to be cointegrated closer to the recording sites, in turn presenting the challenge of creating high-density, yet low-power, active recording probes. ${ }^{11}$

\section{ASSOCIATED CONTENT}

\section{Supporting Information}

The Supporting Information is available free of charge on the ACS Publications website at DOI: 10.1021/acs.nanolett.6b02673. 
Neural probe fabrication; heat dissipation analysis; surgical procedures and in vivo recording methods; histology; recording analysis and validation (PDF)

\section{AUTHOR INFORMATION}

\section{Corresponding Authors}

*E-mail: thanos@caltech.edu.

*E-mail: roukes@caltech.edu.

\section{Author Contributions}

G.R. and E.V.L. contributed equally to this work. System architecture and specification: G.R., E.V.L., M.L.R., A.G.S.; component design: G.R., E.V.L.; component mask layout: G.R., E.V.L.; component fabrication prototyping: G.R., D.C.; component foundry fabrication: G.R., E.V.L., M.L.R., A.G.S.; system packaging: G.R., E.V.L.; system software development: E.V.L.; system benchtop evaluation: G.R., E.V.L.; system in vivo evaluation: G.R., E.V.L., A.G.S.; manuscript preparation and figure creation: G.R., E.V.L. with input from A.G.S., M.L.R., D.C.

\section{Notes}

The authors declare no competing financial interest.

\section{ACKNOWLEDGMENTS}

Foundry fabrication was carried out at CEA/LETI, Grenoble under the aegis of the Alliance for Nanosystems VLSI; we especially thank Denis Renaud, Eric Rouchouze, and Hughes Metras for their help. We thank Jennifer Mok for histological processing of brains from the in vivo experiments. This work was supported by the Mathers Foundation, the Beckman Institute at Caltech, the Moore Foundation, and the NIH (1DP1OD008255/5DP1MH099907).

\section{REFERENCES}

(1) Wise, K. D.; Sodagar, A. M.; Yao, Y.; Gulari, M. N. Proc. IEEE 2008, 96, 1184-1202.

(2) Alivisatos, A. P.; Andrews, A. M.; Boyden, E. S.; Chun, M.; Church, G. M.; Deisseroth, K.; Donoghue, J. P.; Fraser, S. E.; Lippincott-Schwartz, J.; Looger, L. L.; Masmanidis, S.; McEuen, P. L.; Nurmikko, A. V.; Park, H.; Peterka, D. S.; Reid, C.; Roukes, M. L.; Scherer, A.; Schnitzer, M.; Sejnowski, T. J.; Shepard, K. L.; Tsao, D.; Turrigiano, G.; Weiss, P. S.; Xu, C.; Yuste, R.; Zhuang, X. ACS Nano 2013, 7 (3), 1850-1866.

(3) Buzsáki, G.; Stark, E.; Berényi, A.; Khodagholy, D.; Kipke, D. R.; Yoon, E.; Wise, K. D. Neuron 2015, 86 (1), 92-105.

(4) Buzsáki, G. Nat. Neurosci. 2004, 7 (5), 446-451.

(5) Marblestone, A. H.; Zamft, B. M.; Maguire, Y. G.; Shapiro, M. G.; Cybulski, T. R.; Glaser, J. I.; Amodei, D.; Stranges, P. B.; Kalhor, R.; Dalrymple, D. A.; Seo, D.; Alon, E.; Maharbiz, M. M.; Carmena, J. M.; Rabaey, J. M.; Boyden, E. S.; Church, G. M.; Kording, K. P. Frontiers in Computational Neuroscience 2013, 7, 137.

(6) Du, J.; Blanche, T. J.; Harrison, R. R.; Lester, H. A. PLoS One 2011, 6 (10), e26204.

(7) Scholvin, J.; Kinney, J. P.; Bernstein, J. G.; Moore-Kochlacs, C.; Kopell, N.; Fonstad, C. G.; Boyden, E. S. IEEE Trans. Biomed. Eng. 2016, 63 (1), 120-130.

(8) Shobe, J. L.; Claar, L. D.; Parhami, S.; Bakhurin, K. I.; Masmanidis, S. C. J. Neurophysiol. 2015, 114 (3), 2043-2052.

(9) Berenyi, A.; Somogyvari, Z.; Nagy, A. J.; Roux, L.; Long, J. D.; Fujisawa, S.; Stark, E.; Leonardo, A.; Harris, T. D.; Buzsaki, G. J. Neurophysiol. 2014, 111 (5), 1132-1149.

(10) Lopez, C. M.; Andrei, A.; Mitra, S. IEEE J. Solid-State Circuits 2014, 49 (1), 248-261.

(11) Lopez, C. M.; Mitra, S.; Putzeys, J. Solid-State Circuits 2016, 59, 392-393.

(12) Blanche, T. J. J. Neurophysiol. 2005, 93 (5), 2987-3000.
(13) Merriam, M. E.; Dehmel, S.; Srivannavit, O.; Shore, S. E.; Wise, K. D. IEEE Trans. Biomed. Eng. 2011, 58 (2), 397-403.

(14) Merriam, S.; Srivannavit, O. J. Microelectromech. Syst. 2011, 20 (3), 594-600.

(15) Herwik, S.; Kisban, S.; Aarts, A. A. A.; Seidl, K.; Girardeau, G.; Benchenane, K.; Zugaro, M. B.; Wiener, S. I.; Paul, O.; Neves, H. P.; Ruther, P. J. Micromech. Microeng. 2009, 19 (7), 074008-074011.

(16) Najafi, K.; Ji, J.; Wise, K. D. IEEE Trans. Biomed. Eng. 1990, 37 (1), 1-11.

(17) Edell, D. J.; Toi, V. V.; McNeil, V. M.; Clark, L. D. IEEE Trans. Biomed. Eng. 1992, 39 (6), 635-643.

(18) Geddes, L. A.; Roeder, R. Ann. Biomed. Eng. 2003, 31 (7), 879890.

(19) Kotzar, G.; Freas, M.; Abel, P.; Fleischman, A.; Roy, S.; Zorman, C.; Moran, J. M.; Melzak, J. Biomaterials 2002, 23 (13), 2737-2750.

(20) Musallam, S.; Bak, M. J.; Troyk, P. R.; Andersen, R. A. J. Neurosci. Methods 2007, 160 (1), 122-127.

(21) Marin, C.; Fernandez, E. Front. Neuroeng. 2010, 3, 8.

(22) Nicholson, C.; Freeman, J. A. J. Neurophysiol. 1975, 38, 356368.

(23) Karumbaiah, L.; Saxena, T.; Carlson, D.; Patil, K.; Patkar, R.; Gaupp, E. A.; Betancur, M.; Stanley, G. B.; Carin, L.; Bellamkonda, R V. Biomaterials 2013, 34 (33), 8061-8074.

(24) Ferguson, J. E.; Boldt, C.; Redish, A. D. Sens. Actuators, A 2009, 156 (2), 388-393.

(25) Desai, S. A.; Rolston, J. D.; Guo, L.; Potter, S. M. Front. Neuroeng. 2010, 3, 5 .

(26) Loeb, G. E.; Bak, M. J.; Salcman, M.; Schmidt, E. M. IEEE Trans. Biomed. Eng. 1977, 24 (2), 121-128.

(27) Xie, C.; Liu, J.; Fu, T.-M.; Dai, X.; Zhou, W.; Lieber, C. M. Nat. Mater. 2015, 14, 1286.

(28) Jeong, J.-W.; Shin, G.; Park, S. I.; Yu, K. J.; Xu, L.; Rogers, J. A. Neuron 2015, 86 (1), 175-186.

(29) Fomani, A. A.; Mansour, R. R. Sensors 2010, 1601-1604.

(30) Kozai, T. D. Y.; Langhals, N. B.; Patel, P. R.; Deng, X.; Zhang, H.; Smith, K. L.; Lahann, J.; Kotov, N. A.; Kipke, D. R. Nat. Mater. 2012, 11 (12), 1065-1073.

(31) Mercanzini, A.; Cheung, K.; Buhl, D. L.; Boers, M.; Maillard, A.; Colin, P.; Bensadoun, J.-C.; Bertsch, A.; Renaud, P. Sens. Actuators, A 2008, 143 (1), 90-96. 